\title{
Effects of Acute Alcohol Consumption on Cycling Anaerobic Exercise Performance: A Randomized Crossover Study
}

\author{
Ting-Heng Chou ${ }^{1}$ Ph.D, Chansol Hurr ${ }^{2}$ Ph.D \\ ${ }^{1}$ Center for Regenerative Medicine, The Research Institute at Nationwide Children's Hospital, Columbus, OH, USA, ${ }^{2}$ nntegrative Exercise Physiology Laboratory, \\ Department of Physical Education, Jeonbuk National University, Jeonju, Korea
}

PURPOSE: Some athletes consume alcohol prior to training and sports events, possibly because they believe that alcohol may aid in reducing pain and anxiety, increasing confidence, and promoting aggressiveness. While previous studies have shown that alcohol consumption acutely impairs endurance exercise performance, its effects on anaerobic exercise performance have not been well established.Therefore, this study investigated the effect of a small dose of alcohol on anaerobic exercise performance.

METHODS: In a randomized crossover design, nine healthy and recreationally active males $(n=6)$ and females $(n=3)$ participated in the study. Subjects consumed $0.7 \mathrm{~g}$ of alcohol per kg of lean body mass or a calorie/volume-matched sucrose beverage. Reaction time tests were performed before and 25 minutes after beverage consumption. Following the second reaction time test, subjects performed the 20 -second Wingate anaerobic test (WAnT).

RESULTS: Compared to the sucrose drink, alcohol showed no significant effects on peak power $(p=.903)$, average power $(p=.970)$, percentage power drop during WAnT ( $p=.593)$, or total energy produced during WAnT $(p=.923)$. Moreover, alcohol did not affect the time course of power output during a 20s WAnT ( $p=.999$ for all time points). In addition, reaction time was not significantly different when comparing alcoholic with sucrose drinks at different timepoint (Before; $p=.999$, After; $p=.364$ ).

CONCLUSIONS: Although some athletes consume alcohol prior to engaging in sports events, assuming that it may improve anaerobic exercise performance, the results of the present study show that acute consumption of alcohol does not have effects on anaerobic exercise performance when compared to the control beverage.

Key words: Alcohol, Anaerobic power, Reaction time, Performance, Exercise

\section{INTRODUCTION}

Alcohol, less often referred to by its chemical name ethanol, is a common component of a daily diet and one of the most heavily-consumed drinks worldwide. While it is generally agreed that athletes should refrain from consuming alcohol, dietary surveys have shown that self-reported alcohol consumption by athletes contributes as much as $5 \%$ of their total daily energy intake [1]. The average daily intake of alcohol by athletes is in fact similar to the level consumed by the general population
[2]. It has also been reported that $80.5 \%$ of American college studentathletes consume alcohol [3]. Prior to a report by the American College of Sports Medicine (ACSM) Position Stand [4], alcohol was often considered as having ergogenic properties [5]. Some athletes consume alcohol prior to training and sports events [6-8], possibly because they believe that it may help to reduce pain and anxiety, increase confidence, and promote aggressiveness [9].

The widespread use of alcohol among athletic populations may derive from the lack of conclusive evidence from well-controlled experiments.

Corresponding author: Chansol Hurr, Ph.D Tel +82-63-270-2835 Fax +82-63-270-2850 E-mail chansolhajbnu.ac.kr

*This research was supported by the Research Base Construction Fund Support Program funded by Jeonbuk National University in 2020 . It was also supported by a National Research Foundation of Korea (NRF) grant funded by the Korea government (NRF-2019R1F1A1062693). All experiment procedures were conducted in Jeonbuk National University, Korea.

Keywords Alcohol, anaerobic power, reaction time, performance, exercise

Received 19 Jun 2020 Revised 23 Jul 2020 Accepted 3 Aug 2020

(a) This is an Open Access article distributed under the terms of the Creative Commons Attribution Non-Commercial License (https://creativecommons.org/licenses/by-nc/4.0/) which permits unrestricted non-commercial use distribution, and reproduction in any medium, provided the original work is properly cited. 
Several studies have shown that acute alcohol consumption actually impairs endurance exercise performance such as middle-distance running, 60-minute running, or cycling time trials [10-13]. By contrast, several studies have reported that alcohol has no significant effects on submaximal endurance performance [14], 5-mile running time [15], or peak power during maximal cardiopulmonary exercise test [16]. Therefore, acute alcohol consumption in general provides no beneficial effects and may even have detrimental effects on aerobic endurance exercise.

While numerous studies have examined the effects of alcohol on aerobic exercise performance, investigations of its effects on anaerobic performance are relatively fewer in number. McNaughton and Pierce [11] have reported that alcohol had no significant effect on 100-meter sprint performance. However, several confounding factors other than anaerobic power may also impact 100-meter sprint performance, including motor reaction time, beverages consumed, the timeframe of the assessment itself between beverage consumption and the recording of anaerobic performance, and measurement errors deriving from subtle performance differences. Other research has also observed no change in isokinetic power following alcohol consumption $[10,17,18]$. While an isokinetic dynamometer provides quantitative data on motor performance, using isokinetic power as a means of measuring anaerobic exercise performance may limit translating and applying these findings to real-world and competitive contexts.

Reaction time is an indirect index of the processing speed of central nervous system that executes motor response following sensory stimuli such as auditory and visual stimulation [19]. In many sports that require anaerobic power, reaction time determines the subsequent performance in sports that require quick decision in response to visual stimulus such as basketball, football, and Taekwondo [20], and also decides time record at the beginning phase in sprint running and swimming [21]. Several factors are known to affect reaction time including age and fatigue; however, alcohol consumption is consistently shown to impair reaction time [22]. Therefore, it would be critical to assess reaction time as well as anaerobic exercise capacity; however, no studies to date exist looking at both factors in regard to acute effects of alcohol consumption on anaerobic performance.

In the current study, we applied the Wingate anaerobic test, which provides quantitative anaerobic power analysis that requires more motor control and coordination. Furthermore, we evaluated visual reaction time in response to the alcohol ingestion. We hypothesized that consuming even a small dose of alcohol acutely decreases anaerobic exercise performance, independently of visual motor reaction time.

\section{METHODS}

\section{Ethical Approval}

The Institutional Review Board (IRB) at Jeonbuk National University approved all study procedures and the consent process used in the present study (JBNU 2020-01-004-002). Subjects were given a verbal explanation of all procedures and informed of the purpose of the study and risks involved, and written consent was obtained before it began. The study was conducted in compliance with the provisions of the Helsinki Declaration.

\section{Subjects and Experimental Protocol}

Nine healthy and recreationally active males $(n=6)$ and females $(n=3)$ $(28.5 \pm 0.9$ years, $76.3 \pm 1.9 \mathrm{~kg}, 177.7 \pm 1.6 \mathrm{~cm})$ who recreationally consume alcohol (1-2 times/wk) participated in the study. Subjects were instructed to maintain their regular diet during the participation period and were asked to refrain from strenuous exercise, alcohol, and caffeine consumption during the 24 hour period preceding each test visit. Trials were conducted in the morning following an overnight fast (at least 12 hours).

A schematic of the experimental protocol employed is presented in Fig. 1. Subjects visited the laboratory a total of 5 times, consisting of one orientation visit and four test visits. The purpose of the orientation visit was to minimize potential learning effects. During the first visit, subjects were informed of the overall experimental protocol, the potential risks involved, and the purpose of the study itself. After completion of a written consent form, subjects were familiarized with the high intensity cycle ergometer and measuring reaction time by the ground reaction force system. Whole body mass ( $\mathrm{kg}$ ) and lean body mass (kg) were also measured using an InBody 720 body composition analyzer (InBody Co., CA, USA) during the session (Fig. 1A).

To compare blood alcohol concentration after alcohol or sucrose (control) beverage consumption, and optimize timing of reaction time and anaerobic power assessments, the subsequent two visits (Visits 2 and 3, Fig. 1B) were completed in randomized order with at least a 72-hour separation. During Visits 2 and 3, subjects were rested in a sitting position for 5 minutes and consumed alcohol or the sucrose (control) beverage within the next 5 minutes, after which blood alcohol content (BAC) was measured using a digital alcohol detector (breathalyzer AT868, Greenwon, China), and at 15, 25, 35, and 45 minutes after drink inges- 


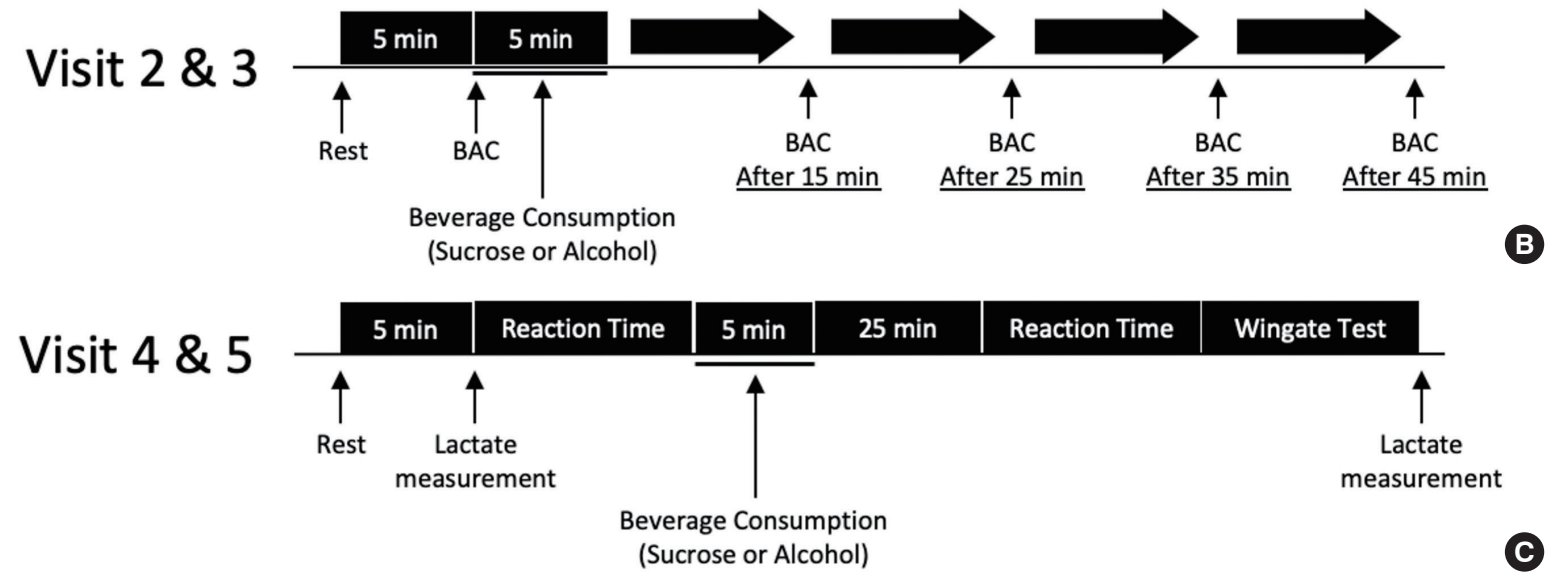

Fig. 1. Study design. During Visit 1 (A), subjects were familiarized with the high intensity cycle ergometer and measuring reaction time by the ground reaction force system. (B) shows indirect assessment of blood alcohol content (BAC) via breathalyzer during Visit 2 and 3 . During Visit 4 and 5 (C), anaerobic exercise capacity was measured following sucrose or alcohol beverage consumption in randomized crossover order. Lactate represents blood sample collection for assessment of blood lactate concentration.

tion. Alcohol and sucrose beverages were calorie-volume matched. Subjects consumed $0.7 \mathrm{~g}$ of alcohol per $\mathrm{kg}$ of lean body mass with water added up to $300 \mathrm{~mL}$ [17]. The composition of the sucrose beverage was based on the calories of alcohol consumed, also with water added up to $300 \mathrm{~mL}$.

Based on the data that we collected during Visits 2 and 3, it was found that $\mathrm{BAC}$ reached its peak 25 minutes after alcohol ingestion and was maintained at a relatively constant level for 45 minutes after alcohol consumption (Fig. 2). Accordingly, reaction time tests were performed 25 minutes after drink ingestion, which was immediately followed by the Wingate anaerobic power assessment (Visit 4 and 5, Fig. 1C). During Visit 4 and 5, subjects rested for 5 minutes, blood samples were collected using standard lancets and disposable strips, and analyzed for blood lactate levels using a portable blood lactate monitoring device (AccuTrend Plus, Roche, Germany). After the lactate measurement, baseline reaction time was evaluated twice using a light-ground reaction force system interface. This was followed by alcohol or sucrose beverage consumption within the next 5 minutes. Reaction time was again assessed 25 minutes following beverage ingestion. Lastly, subjects performed the 20-second Wingate anaerobic test (WAnT), followed by blood lactate measurement. Neither food nor water intake was permitted during implementation of the protocol. All other procedures were identical between the two visits (i.e., Visits 4 and 5).

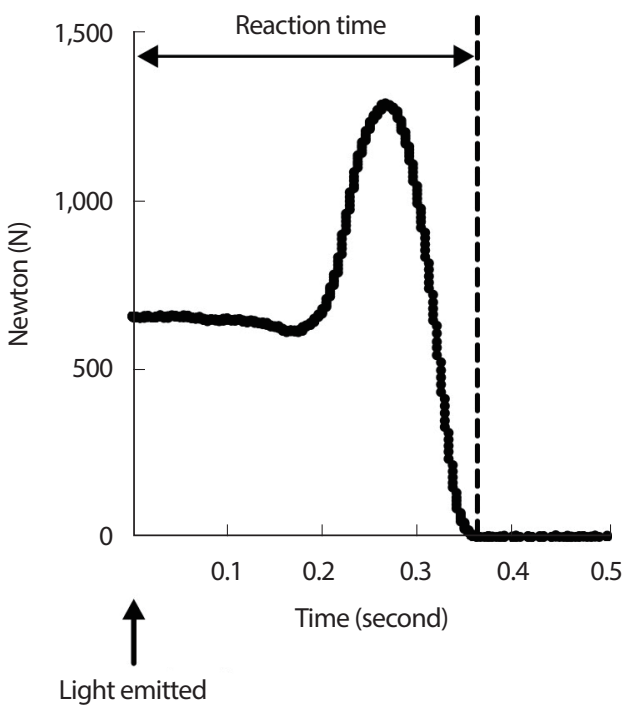

Fig. 2. Ground reaction force (GRF) during reaction time test. Once the diode emits red right, GRF recording begins and reaction time is calculated as shown in Fig. 2.

\section{Wingate Anaerobic Test (WAnT)}

A cycle ergometer (Monark 894E, Vansbro, Sweden) was used for each WAnT. For each subject the saddle height was adjusted so that the knee angle remained at approximately $170^{\circ}$. Subjects' feet were secured to the pedals with foot straps. The cycle ergometer was connected to a PC for data collection and data were analyzed using Monark anaerobic test software. Subjects were instructed to begin pedaling before resistance 
was applied (75 g per kg of body weight). Once subjects reached a pedaling speed of $150 \mathrm{rpm}$, the basket automatically dropped, and subjects began to pedal until exhaustion for 20 seconds. For the purposes of the present study, a modified 20-second WAnT rather than the regular 30-second WAnT was performed, in order to avoid potential stomach upset as well as other risks in response to a WAnT following alcohol consumption. All subjects were given standardized verbal encouragement during the test and instructed to remain seated while the test was in progress.

\section{Reaction Time}

A subject was instructed to stand on a force platform with both feet shoulder-width apart (Advanced Mechanical Technology, Inc., MA, USA). A light emitting diode was placed approximately 3 meters in front of subjects at chest level. As soon as an experimenter pushed start button, the diode emitted red light and, simultaneously, the software (KwonGRF, VISOL Inc., Korea) began recording GRF. The subject was instructed to step both feet off from the force platform in the lateral directions once the diode emitted the light. Two reaction times were averaged in order to minimize trial-by-trial variability. Based on the changes in GRFs (Fig. 2), we determined the reaction times for subjects to escape from the force platform following visual recognition of the light. This should be an index of neuronal reactivity from the visual stimulus to motor neuronal control [23]. GRFs were collected at 1,200 Hz and lowpass-Butterworth-filtered at $6 \mathrm{~Hz}$ [24].

\section{Statistical Analysis}

Data are expressed as the mean \pm SE. Multiple group comparisons were performed using two-way repeated measures analysis of variance (ANOVA) ([before/after $] \times[$ sucrose/alcohol] or $[$ time $] \times[$ sucrose/alcohol $]$ ), followed by Bonferroni post hoc analysis. Unpaired Student's $t$-test was used for variables in anaerobic power output. Significance was set at $p<.05$ (Prism 8.3, GraphPad).

\section{RESULTS}

\section{Alcohol Concentration in Blood}

Blood alcohol concentration (BAC) reached peak value $(0.03 \pm 0.01 \%)$ 25 minutes after alcohol consumption and was maintained at a relatively constant level for 45 minutes. Additionally, no blood alcohol was detected after sucrose beverage consumption by all subjects. BAC was signifi-

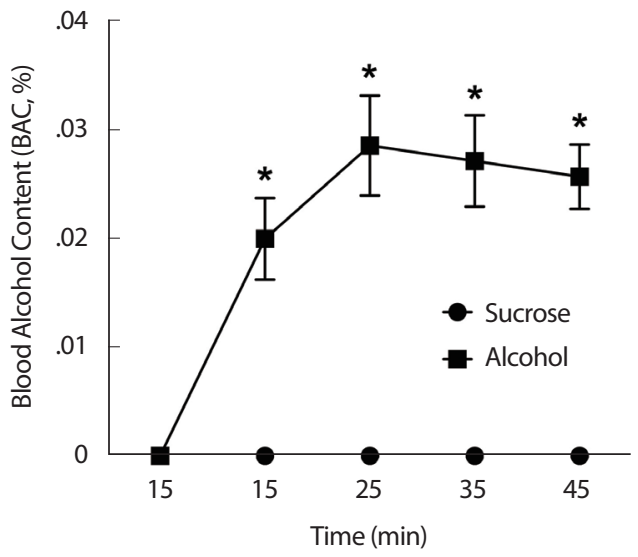

Fig. 3. Blood Alcohol Content (BAC) test. BAC reached its peak at 25 minutes following alcohol consumption and was maintained for 45 minutes. No detectable BAC was found in sucrose consumption. Values represent mean \pm SE. ${ }^{*} p<.05$ vs. baseline.

cantly higher after alcohol compared to sucrose consumption $(p<.001)$. $\mathrm{BAC}$ responses after consumption of alcohol or sucrose beverages are presented in Fig. 3.

\section{Wingate Anaerobic Test}

Power achieved during the WAnT was presented and expressed as Watts per lean body mass (W/kg). Compared to the sucrose drink, alcohol had no significant effect on peak power (Fig. 4A, sugar $8.53 \pm 0.70$ vs. alcohol $8.65 \pm 0.62 \mathrm{~W} / \mathrm{kg} ; p=.903$ ), average power (Fig. 4B, $6.70 \pm 0.46$ vs $6.73 \pm 0.42 \mathrm{~W} / \mathrm{kg} ; p=.970$ ), power drop during WAnT (Fig. 4C, sugar 47.1 \pm 7.9 vs. alcohol $48.0 \pm 4.1 \%$; $p=.593$ ), or total energy produced during WAnT (Fig. 4D, 10,249 $\pm 1,221$ vs. 10,417 $\pm 1,196$ Joules; $p=.923$ ). In addition, alcohol did not affect the time course of power output during the 20 seconds WAnT (Fig. 4E, $p=.999$ for all four time points). All other variables during WAnT including time to peak power, time at maximal speed, minimum power ( $\mathrm{W}$ and $\mathrm{W} / \mathrm{kg}$ ), power drop $(\mathrm{W})$ were not different between sucrose and alcohol beverage consumption (data not shown).

\section{Reaction Time}

Reaction time was not significantly altered by consumption of either alcohol (Fig. $5 \mathrm{~A}, 0.371 \pm 0.017$ vs. $0.353 \pm 0.012$ seconds; $p=.886$ ) or sucrose $(0.382 \pm 0.017$ vs. $0.386 \pm 0.018$ seconds; $p=.999)$. There were no significant differences between reaction times after consuming alcohol or sucrose drinks at any timepoint (Before, sugar $0.382 \pm 0.017$ vs alcohol $0.371 \pm 0.017 \mathrm{~s} ; p=0.999$, After, sugar $0.386 \pm 0.018$ vs. alcohol $0.353 \pm 0.012$ second; $p=.364$ ). Reaction times before and after alcohol or sucrose con- 
Peak power

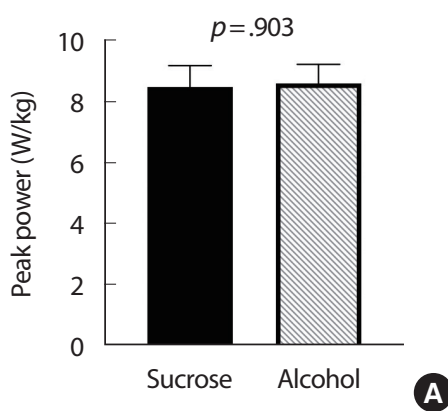

Power drop

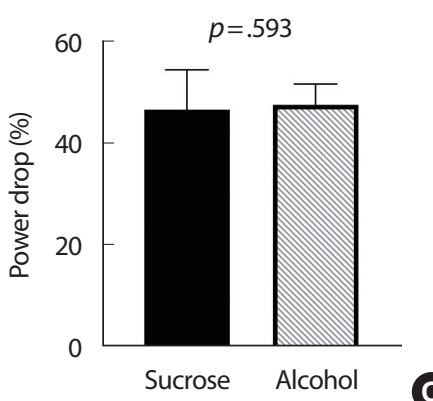

Average power
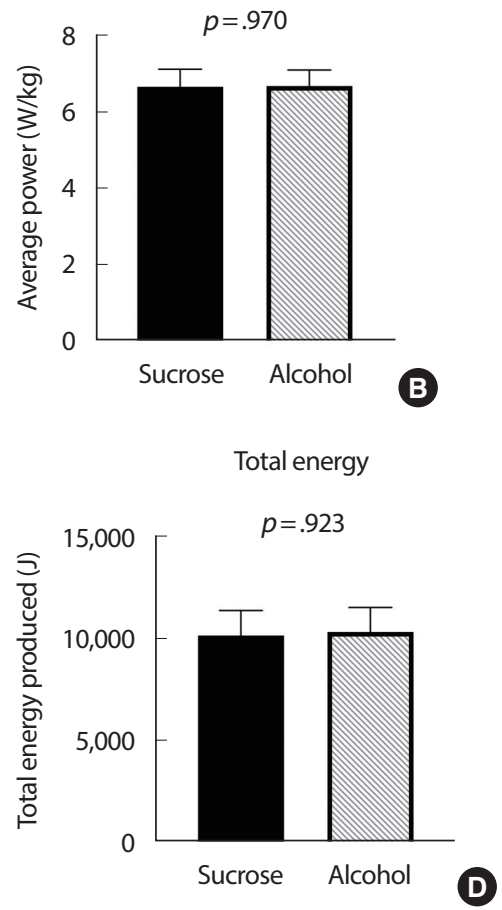

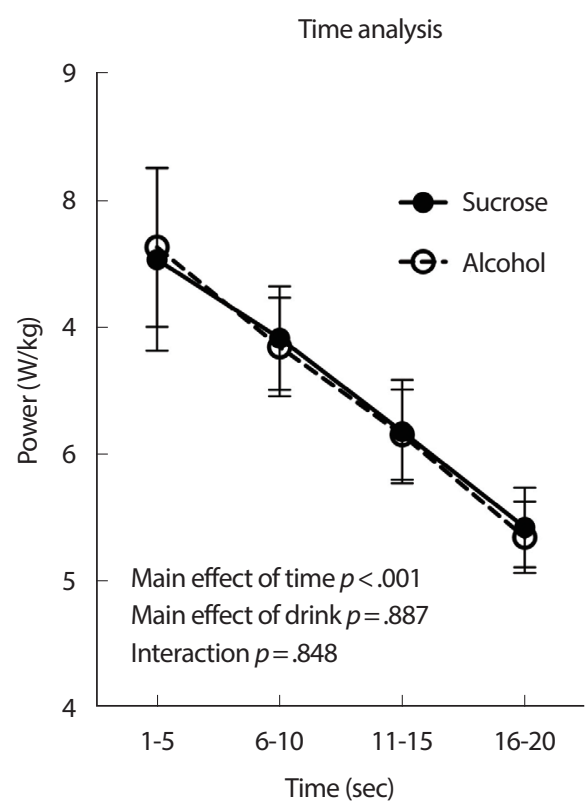

E

Fig. 4. Power analysis from 20-second Wingate test. Peak power (A), average power (B), power drop (C), total energy produced (D), and time analysis during 20-second Wingate test $(E)$ are shown. Values represent mean \pm SE.
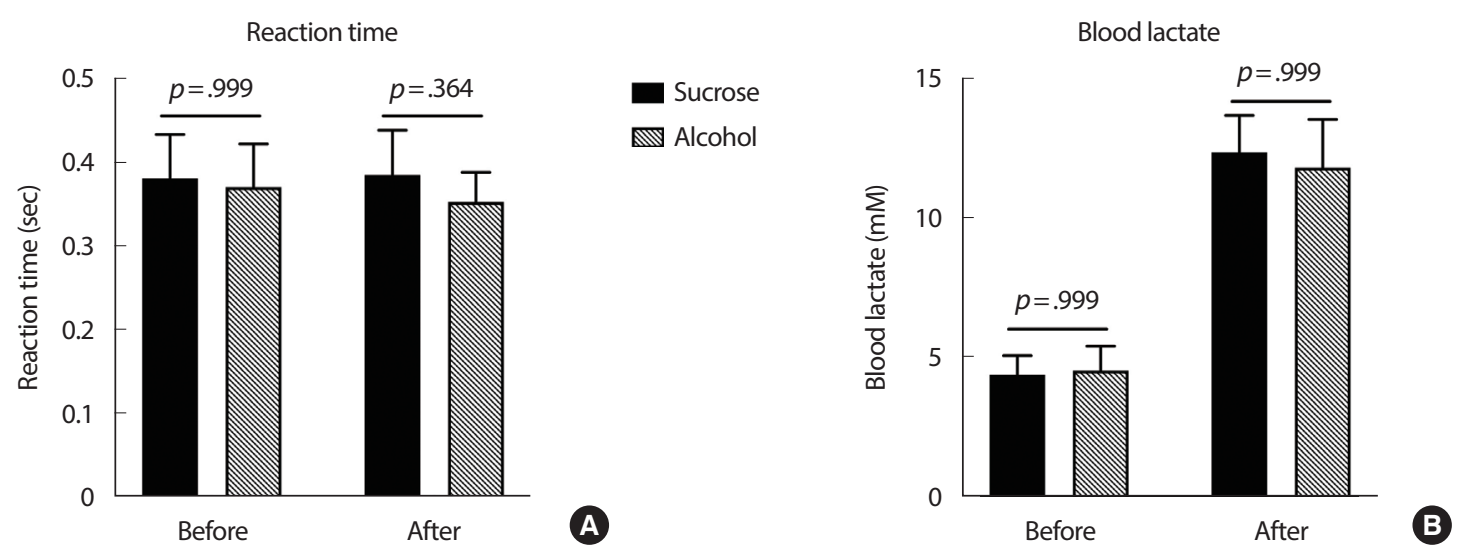

Fig. 5. Reaction time (A) and blood lactate (B). No difference was found between sucrose and alcohol consumption for either variable. Values represent mean \pm SE. ${ }^{*} p<.05$ vs. baseline.

sumption are presented in Fig. 5A.

\section{Blood Lactate}

Baseline blood lactate concentration was similar at baseline before alcohol or sucrose beverage consumption (Fig. $5 \mathrm{~B}, 4.4 \pm 0.7$ vs. $4.6 \pm 0.9$ $\mathrm{mM} ; p=.999)$. Blood lactate concentration significantly increased after WAnT with either alcohol or sucrose consumption ( $p<.001$ vs. baseline). However, blood lactate concentrations after WAnT were not significantly different for either condition (sugar $12.4 \pm 1.3$ vs. alcohol $11.8 \pm 1.7 \mathrm{mM}$; $p=.999$ ). Blood lactate concentrations at baseline and after WAnT with either alcohol or sucrose beverage consumption are presented in Fig. 5B.

\section{DISSCUSSION}

While the effects of acute alcohol consumption on aerobic exercise performance have been investigated in numerous studies [10-13], its ef- 
fect on anaerobic performance remains far less understood [10,11,17]. The present study showed that a small dose of alcohol ( $0.7 \mathrm{~g}$ per lean body mass) consumption had no acute effect on anaerobic exercise performance indexed by peak power, average power, power drop, and total energy produced during a 20 -seconds WAnT, independently of visualmotor reaction time.

Our findings accord with those of previous studies that have investigated the effects of acute alcohol consumption on anaerobic exercise performance, although the metrics of anaerobic exercise performance are different in each case. For example, previous studies have shown that isokinetic power does not change following alcohol consumption $[10,17,18]$. However, isokinetic power primarily involves motor performance and is less applicable to real-world anaerobic performance. The present study has further demonstrated that acute alcohol consumption does not affect anaerobic performance requiring greater motor control and coordination, as assessed by the cycling Wingate test. Four functional muscle groups are associated in cycling including the hip and knee extensors, the plantar flexors and hip extensors, hip and knee flexors, and ankle dorsiflexors and hip flexors [25]. During downstroke, two functional extensor groups provide energy while the other two flexor groups enable upstroke [26]. Importantly, the activity of Central Pattern Generators (CPG) in the spinal cord is associated with rhythmic motor activity and motor coordination of those functional muscle groups during cycling [27]. Thus, anaerobic performance assessed by the Wingate test in the current study provides more applicable insight.

Additionally, McNaughton and Preece [11] have reported that alcohol did not affect 100-meter sprint performance when blood alcohol concentration was as high as $0.1 \mathrm{mg} / \mathrm{mL}$ (i.e., $0.01 \% \mathrm{BAC}$ ). However, in their experiment alcohol consumption may have delayed motor reaction time, a critical component in 100-meter sprint performance [11]. Therefore, the possibility remains that improved anaerobic power performance due to alcohol consumption may be concealing a delayed reaction in the starting phase of the sprint. The present study, however, confirmed that visual-motor reaction time was not altered at peak BAC $(0.03 \pm 0.01 \%)$ before the WAnT, confirming that acute alcohol consumption did not affect anaerobic exercise performance, independently of visual-motor reaction time. Furthermore, the control beverages used in previous studies that investigated the acute effects of alcohol on anaerobic performance were not calorie-volume matched with the experimental alcohol beverages [11,17], which could be a nutritional/metabolic confounding factor for power performance $[28,29]$. Altogether, the present study demon- strated that a small dose of alcohol did not affect anaerobic exercise, which is consistent with the findings of previous studies [11,17]. More importantly, our study improved the application of these findings by addressing some of the limitations of previous studies.

The present study observed that an acute small dose of alcohol affected neither resting nor post-WAnT blood lactate concentrations. Alcohol has been shown to reduce liver gluconeogenesis from lactate in perfused rat liver [30]. Additionally, elevated resting lactate to pyruvate ratios has also been observed after infusion or ingestion of alcohol in healthy subjects [31]. This observation is likely induced by the activation of alcohol dehydrogenase enzyme (ADH) when a low concentration of alcohol is presented. The oxidation of ethanol by ADH elevates the ratio of nicotinamide adenine dinucleotide (NADH) to NAD+, leading to an increase in lactate/pyruvate ratio [32-34]. The similar resting and postWAnT blood lactate concentrations after alcohol consumption compared to sucrose drink consumption in the present study might stem from the fact that we compared blood lactate concentration rather than measuring the lactate/pyruvate ratio. Comparing the lactate/pyruvate ratio may reveal differences between the alcohol and sucrose drink conditions. In addition, post-WAnT blood lactate concentration was primarily determined by the rate of exercising muscle glycolysis and was less influenced by liver lactate metabolism.

\section{Limitations}

The present study has some limitations. Only a relatively small dose of alcohol ( 0.7 g per lean body mass) was investigated, which had no significant effect on WAnT anaerobic exercise performance. Whether larger doses of alcohol would produce similar results remains unknown. We elected to investigate the effects of a small dose of alcohol because we also wanted to control for reaction time, as was achieved in the present study. Additionally, while acute alcohol consumption has been shown as having no significant effects on anaerobic exercise performance, since chronic alcohol consumption has been shown to have detrimental effects on skeletal and cardiac muscles [35-37], metabolic recovery [38], post-exercise glycogen resynthesis [39], and immune function [40] it might also be hypothesized as impairing anaerobic exercise performance. We also analyzed sex differences in anaerobic power outputs following acute alcohol ingestion. Although there was no discrepancy between males and females (data not shown) in response to acute alcohol consumption, future research is needed to investigate sex differences as sample size in the present study was too small (6 males and 3 females). 
Furthermore, since all of the subjects in the current study were recreationally active and regular consumers of alcohol, further study is necessary to investigate how far the findings of the present study are applicable to different populations, such as trained athletes or people with different habits of alcohol consumption.

\section{Conclusion}

Although some athletes consume alcohol prior to engaging in sports events, assuming that it may improve anaerobic exercise performance, the results of the present study show that a small dose of alcohol has no effects on anaerobic exercise performance, assessed by a 20-second WAnT, nor on blood lactate response to the WAnT. In addition, this absence of any beneficial effect of alcohol on anaerobic exercise performance was observed to be independent of reaction time. Further investigation is warranted to explore the chronic effects of alcohol consumption on anaerobic exercise performance.

\section{CONFLICT OF INTEREST}

No potential conflict of interest relevant to this article was reported.

\section{ACKNOWLEDGMENTS}

The authors would like to express our appreciation to all of our participants for their participation in this study.

\section{REFERENCES}

1. Maughan RJ, Burke LM, Alcohol and sport, in Handbook of Sports Medicine and Science Sports Nutrition, RJ Maughan, Editor, Blackwell Science: Malden, Massachusetts 2002. p. 64-70.

2. Gutgesell ME, Timmerman M, Keller A. Reported alcohol use and behavior in long-distance runners. Med Sci Sports Exerc. 1996;28 (8):1063-70.

3. Green GA, Uryasz FD, Petr TA, Bray CD. NCAA study of substance use and abuse habits of college student-athletes. Clin J Sport Med. 2001;11(1):51-6.

4. American College of Sports Medicine. Alcohol use in sports. Med Sci Sports Exerc. 1982;14(6):ix-xi.

5. Eichner ER. Ergolytic drugs in medicine and sports. Am J Med. 1993;94(2):205-11.
6. Maughan RJ. Alcohol and football. J Sports Sci. 2006;24(7):741-8.

7. Gutgesell M, Canterbury R. Alcohol usage in sport and exercise. Addict Biol. 1999;4(4):373-83.

8. Pesta DH, Angadi SS, Burtscher M, Roberts CK. The effects of caffeine, nicotine, ethanol, and tetrahydrocannabinol on exercise performance. Nutr Metab (Lond). 2013;10(1):71.

9. Katch VL, McArdle WD, Katch FI. Nutritional and Pharmacologic Aids to Performance, in Essentials of Exercise Physiology, Lippincott Williams \& Wilkins: Baltimore, MD; 2011. p. 109-48.

10. O'Brien CP. Alcohol and sport. Impact of social drinking on recreational and competitive sports performance. Sports Med. 1993;15 (2):71-7.

11. McNaughton L, Preece D. Alcohol and its effects on sprint and middle distance running. Br J Sports Med. 1986;20(2):56-9.

12. Kendrick ZV, Affrime MB, Lowenthal DT. Effect of ethanol on metabolic responses to treadmill running in well-trained men. J Clin Pharmacol. 1993;33(2):136-9.

13. Lecoultre V, Schutz Y. Effect of a small dose of alcohol on the endurance performance of trained cyclists. Alcohol Alcohol. 2009;44(3):27883.

14. Bond V, Franks BD, Howley ET. Effects of small and moderate doses of alcohol on submaximal cardiorespiratory function, perceived exertion and endurance performance in abstainers and moderate drinkers J Sports Med Phys Fitness. 1983;23(2):221-8.

15. Houmard JA, Langenfeld ME, Wiley RL, Siefert J. Effects of the acute ingestion of small amounts of alcohol upon 5-mile run times. J Sports Med Phys Fitness. 1987;27(2):253-7.

16. Popovic D, Damjanovic SS, Plecas-Solarovic B, Pesic V, Stojiljkovic S, et al. Exercise capacity is not impaired after acute alcohol ingestion: a pilot study. J Cardiovasc Med (Hagerstown). 2016;17(12):896-901.

17. Poulsen MB, Jakobsen J, Aagaard NK, Andersen H. Motor performance during and following acute alcohol intoxication in healthy nonalcoholic subjects. Eur J Appl Physiol. 2007;101(4):513-23.

18. Clarkson PM, Reichsman F. The effect of ethanol on exercise-induced muscle damage. J Stud Alcohol. 1990;51(1):19-23.

19. Woods DL, Wyma JM, Yund EW, Herron TJ, Reed B. Factors influencing the latency of simple reaction time. Front Hum Neurosci. 2015; 9:131.

20. Atan Tl, Akyol P. Reaction times of different branch athletes and correlation between reaction time parameters. Procedia Social and Behavioral Sciences. 2014;116:2886-9. 
21. Morin JB, Bourdin M, Edouard P, Peyrot N, Samozino P, et al. Mechanical determinants of 100-m sprint running performance. Eur J Appl Physiol. 2012;112(11):3921-30.

22. Tzambazis K, Stough C. Alcohol impairs speed of information processing and simple and choice reaction time and differentially impairs higher-order cognitive abilities. Alcohol Alcohol. 2000;35(2):197-201.

23. Spiteri T, Cochrane JL, Nimphius S. The evaluation of a new lowerbody reaction time test. J Strength Cond Res. 2013;27(1):174-80.

24. Kim S, Lockhart T, Yoon HY. Relationship between age-related gait adaptations and required coefficient of friction. Saf Sci. 2005;43 (7):425-36.

25. Raasch CC, Zajac FE, Ma B, Levine WS. Muscle coordination of maximum-speed pedaling. J Biomech. 1997;30(6):595-602.

26. Raasch CC, Zajac FE. Locomotor strategy for pedaling: muscle groups and biomechanical functions. J Neurophysiol. 1999;82(2):515-25.

27. Driss T, Vandewalle H. The measurement of maximal (anaerobic) power output on a cycle ergometer: a critical review. Biomed Res Int. 2013;2013:589361.

28. Lee JD, Sterrett LE, Guth LM, Konopka AR, Mahon AD. The effect of pre-exercise carbohydrate supplementation on anaerobic exercise performance in adolescent males. Pediatr Exerc Sci. 2011;23(3):344-54.

29. Krings BM, Peterson TJ, Shepherd BD, McAllister MJ, Smith JW. Effects of Carbohydrate Ingestion and Carbohydrate Mouth Rinse on Repeat Sprint Performance. Int J Sport Nutr Exerc Metab. 2017;27 (3):204-12.

30. Krebs HA. The effects of ethanol on the metabolic activities of the liver. Adv Enzyme Regul. 1968;6:467-80.

31. Volpi E, Lucidi P, Cruciani G, Monacchia F, Reboldi G, et al. Nicotin- amide counteracts alcohol-induced impairment of hepatic protein metabolism in humans. J Nutr. 1997;127(11):2199-204.

32. Forsander OA. Influence of ethanol on the redox state of the liver. Q J Stud Alcohol. 1970;31(3):550-70.

33. Lundquist F, Tygstrup N, Winkler K, Mellemgaard K, Munck-Petersen S. Ethanol metabolism and production of free acetate in the human liver. J Clin Invest. 1962;41:955-61.

34. Tygstrup N, Winkler K, Lundquist F. The Mechanism of the Fructose Effect on the Ethanol Metabolism of the Human Liver. J Clin Invest. $1965 ; 44: 817-30$

35. Richardson P, McKenna W, Bristow M, Maisch B, Mautner B, et al. Report of the 1995 World Health Organization/International Society and Federation of Cardiology Task Force on the Definition and Classification of cardiomyopathies. Circulation. 1996;93(5):841-2.

36. Trounce I, Byrne E, Dennett X. Biochemical and morphological studies of skeletal muscle in experimental chronic alcoholic myopathy Acta Neurol Scand. 1990;82(6):386-91

37. Vila L, Ferrando A, Voces J, Cabral de Oliveira C, Prieto JG, et al. Effect of chronic ethanol ingestion and exercise training on skeletal muscle in rat. Drug Alcohol Depend. 2001;64(1):27-33.

38. Maughan RJ BL. Handbook of sports medicine and science: sports nutrition. Oxford: Blackwell Sciences Ltd. 2002.

39. Burke LM, Collier GR, Broad EM, Davis PG, Martin DT, et al. Effect of alcohol intake on muscle glycogen storage after prolonged exercise. J Appl Physiol (1985). 2003;95(3):983-90.

40. Pallister I. Current concepts of the inflammatory response after major trauma: an update. Injury. 2005;36(1):227-9; author reply. 229-30. 